\title{
Non-linear model predictive control of conically shaped liquid storage tanks
}

\author{
Martin Klaučo, Luboš Čirka, \\ Juraj Kukla \\ Institute of Information Engineering, Automation, and Mathematics, \\ Faculty of Chemical and Food Technology, Slovak University of Technology in Bratislava, \\ Radlinského 9, SK-812 37 Bratislava, Slovak Republic \\ martin.klauco@stuba.sk
}

\begin{abstract}
This paper deals with the analysis and design of a model predictive control (MPC) strategy used in connection with level control in conically shaped industrial liquid storage tanks. The MPC is based on a non-linear dynamic model describing changes of the liquid level concerning changes in the inlet flow of the liquid. Euler discretization of the dynamic system was applied to transform con-tinuous time dynamics to its discrete-time counterpart used in non-linear MPC (NMPC) design. By means of a simulation case study, NMPC has been shown to track the changes of the liquid level, hence provides increased control performance. This paper also compares the traditional approach of optimal control, linear MPC, with the NMPC strategy.
\end{abstract}

\section{Introduction}

Model predictive control is a well-established control strategy in chemical process control. The main advantages stem from optimal shaping of the trajectory of manipulated variables with respect to the performance criteria and technological and safety constraints (Mayne et al., 2000; Camacho and Bordons, 2007). Optimal control strategies have been systematically addressed in countless scientific works, including time optimal control (Sharma et al., 2015) or standard model predictive control (Muske and Badgwell, 2002; Kvasnica et al., 2010; Bakošová and Oravec, 2014). All aforementioned works, however, focus on the standardized design of model predictive control which relies on linear state space models of the controlled plant. Such approaches introduce an obstacle called "model-mismatch", where the design model in the controller does not match the actual process.

To remedy the situation, researchers focus on non-linear model predictive control (NMPC) which improves the given control strategies by incorporating non-linear equations of the system dynamics (Allgöwer et al., 2004). This work focuses on the application of such a controller to the most common chemical process, the control of a level of the liquid inside storage tanks. Specifically, we focused on a conically-shaped liquid storage tank.

This paper is organized as follows. First, the nonlinear mathematical model of the conical tank is introduced. Second, the design of two controllers: linear MPG and non-linear MPG, are considered. And then, the performance of designed control- lers by means of a simulation case study is compared.

\section{Mathematical Modeling of Conically Shaped Tanks}

The dynamic mathematical model of a tank with one inlet stream, denoted as $q_{\text {in }}(t)$, and one outlet stream, expressed by $q_{\text {out }}(t)$, is given by a mass balance equation of the following form

$$
q_{\text {in }}(t)=q_{\text {out }}(t)+\frac{\mathrm{d} V(t)}{\mathrm{d} t},
$$

where $V(t)$ stands for the liquid volume inside the tank. In this work, the level of the liquid inside the tank was considered as a process variable. Thus, the model in Eq. (1) is rewritten to

$$
q_{\text {in }}(t)=k_{\mathrm{v}} \sqrt{h(t)}+\frac{\mathrm{d} V(t)}{\mathrm{d} h} \frac{\mathrm{d} h(t)}{\mathrm{d} t}
$$

and $F(h)$ is defined as

$$
F(h)=\frac{\mathrm{d} V(t)}{\mathrm{d} h} .
$$

For the purpose of simulations, the model in Eq. (2) is converted to a non-linear state space form

$$
\frac{\mathrm{d} h(t)}{\mathrm{d} t}=\frac{1}{F(h)}\left(q_{\text {in }}(t)-k_{\mathrm{v}} \sqrt{h(t)}\right) .
$$

Variable $k_{v}$ corresponds to an output valve coefficient, which can be derived from the Bernoulli equation and it represents the friction of liquid movement in the outlet pipe (Mikleš and Fikar, 2007, ch. 2).

Here, a controller design based on a discrete time model is considered to obtain the non-linear system 
model by Euler discretization of Eq. (4). Specifically,

$$
h\left(t+T_{\mathrm{s}}\right)=h(t)+T_{\mathrm{s}} \cdot\left(\frac{1}{F(h)}\left(q_{\text {in }}(t)-k_{\mathrm{v}} \sqrt{h(t)}\right)\right),
$$

where variable $T_{\mathrm{s}}$ represents the sampling time. Even though the Euler discretization process can be inexact, it is often used in the controller design as suggested by Lawryńczuk (2017).

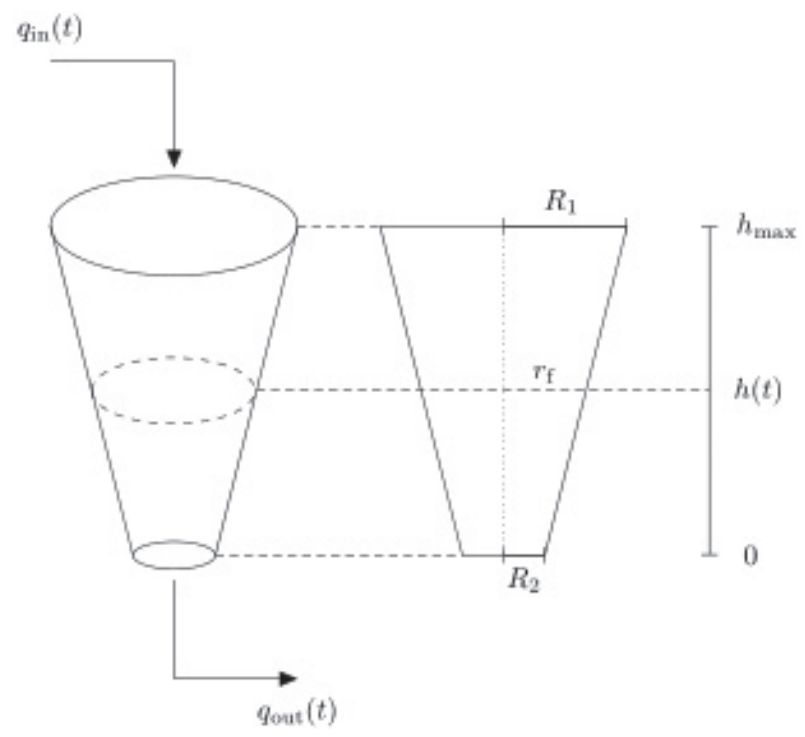

Fig. 1. Illustration of the conically-shaped tank.

An inverted frustum of a right cone was considered as an open conical tank. Geometrical representation of the conical tank is shown in Fig. 1. Mathematical model of such a process was derived based on the findings of King (2010). Concretely, volume of the frustum was expressed as a function of the liquid level. The tank is characterized by variables $R_{1}, R_{2}$, which represent the radii of the bottom and upper base, respectively, and by the height, $h_{\max }$ (cf. Fig. 1). The liquid volume inside the frustum is given by

$$
V_{\mathrm{f}}(h(t))=\frac{\pi h(t)}{3}\left(r_{\mathrm{f}}^{2}(h(t))+R_{2} r_{\mathrm{f}}(h(t))+R_{2}^{2}\right),
$$

where variable $r_{\mathrm{f}}(h(t))$ is the radius of a disc representing the surface of the liquid at level $h(t)$. Radius $r_{\mathrm{f}}(h(t))$ is an explicit function of the liquid level and is expressed as

$$
r_{\mathrm{f}}(h(t))=R_{2}+\frac{R_{1}-R_{2}}{h_{\max }} h(t) .
$$

Then, equuations (7) and (6) were combined to obtain the liquid volume as a function of the level, $h(t)$. The liquid volume is then given as

$$
\begin{gathered}
V_{\mathrm{f}}(h(t))=\frac{\pi h(t)}{3} \times \\
\times\left(3 R_{2}^{2}+3 R_{2} \frac{R_{1}-R_{2}}{h_{\text {max }}} h(t)+\left(\frac{R_{1}-R_{2}}{h_{\text {max }}}\right)^{2} h^{2}(t)\right) .
\end{gathered}
$$

The final non-linear state space model was derived from the expressions for volume, $V_{\mathrm{f}}$, and the general mass balance model in (2). The specific dynamics is then in the form

$$
q_{\text {in }}(t)=k_{\mathrm{v}} \sqrt{h(t)}+\pi\left(R_{2}+h(t) \frac{R_{1}-R_{2}}{h_{\text {max }}}\right)^{2} \frac{\mathrm{d} h(t)}{\mathrm{d} t} .
$$

Symbols and physical quantities used in the previous derivations are reported in Table 1 . The non-linear mathematical model presented in Eq. (9) is then used to design the NMPC strategy addressed in the next section.

Tab. 1. Parameters of the conical tank system and the related system dynamics.

\begin{tabular}{lcc}
\hline Physical quantity & Symbol & Value \\
\hline Height steady state & $h_{\mathrm{L}}$ & $0.4 \mathrm{~m}$ \\
Inlet steady state & $q_{\text {in, } \mathrm{L}}$ & $0.0474 \mathrm{~m}^{3} \mathrm{~s}^{-1}$ \\
Valve coefficient & $k_{\mathrm{V}}$ & $0.075 \mathrm{~m}^{2.5} \mathrm{~s}^{-1}$ \\
Maximum height & $h_{\max }$ & $2 \mathrm{~m}$ \\
Upper radius & $R_{1}$ & $1 \mathrm{~m}$ \\
Bottom radius & $R_{2}$ & $0.4 \mathrm{~m}$ \\
Minimum flow & $q_{\text {in, } \min }$ & $0 \mathrm{~m}^{3} \mathrm{~s}^{-1}$ \\
Maximum flow & $q_{\text {in, } \max }$ & $0.2 \mathrm{~m}^{3} \mathrm{~s}^{-1}$ \\
Sampling time & $T_{\mathrm{S}}$ & $2 \mathrm{~s}$ \\
\hline
\end{tabular}

\section{Design of Controllers}

In this work, the design of a non-linear model predictive control strategy, which exploits the nonlinear nature of the dynamic model is considered. To demonstrate the benefits of the non-linear controller, this approach was compared with the standardized linear version of MPC. Both these controllers are implemented in the scheme depicted in Fig. 2.

The control loop was extended with a state observer to estimate the effect of model mismatch and disturbances. Such control strategy is standard practice in linear-based MPC as suggested by Rawlings and Mayne (2009) and Muske (1997). The linear Kalman Filter or Luenberger observer were used to obtain the estimations. However, in case of the NMPC strategy, the observer in form of extended Kalman filter, which is often used in time optimal control problems (Jelemenský et al., 2016), was constructed. 
The extended Kalman filter dynamics is given by

$$
\begin{gathered}
\hat{x}_{k}=f\left(x_{k}, u_{k}\right), \\
P_{k}=F_{k} P_{k}+P_{k} F_{k}^{\mathrm{T}}+Q,
\end{gathered}
$$

where the actual estimation of signals takes place in discrete time with the observer update calculated as

$$
\begin{gathered}
L_{k}=P_{k} C^{\mathrm{T}}\left(C P_{k} C^{\mathrm{T}}+R\right), \\
\hat{x}_{k}=\hat{x}_{k-1}+L_{k}\left(h_{\mathrm{m}, k}-\hat{x}_{k-1}\right), \\
P_{k+1}=\left(I-L_{k} C_{k}\right) P_{k},
\end{gathered}
$$

where $\hat{x}$ denotes the estimated state variable. Then, the filter dynamic equation (10a) is equal to the system dynamics given by Eq. (5), while matrix $P$ denotes the covariance matrix. State transition matrix $F$ is defined as Jacobian, namely

$$
F_{k}=\left.\frac{\partial f(x, u)}{\partial x}\right|_{x_{k}, u_{k}} .
$$

Note that since the state variable is equal to the measured output, denoted as $y_{\mathrm{k}}, C_{\mathrm{k}}$ is equal to $I$. Matrices $Q$ and $R$ can be considered as tuning parameters of the extended Kalman filter and they should be chosen with respect to the stochastic properties of the state and measurement noise, respectively. Variable $L_{\mathrm{k}}$ denotes the time-varying gain of the Kalman filter.

It is important to note that the estimated state variable $\hat{x}$ is extended by an unmeasured disturbance which enforces an off-set free control demonstrated by simulation case studies below.

Structure of the state observed remains the same for the linear MPC strategy as for the NMPC one. However, the nonlinear model in Eq. (10a) was replaced with the linear state space representation of the model and matrix $F$ was constant.

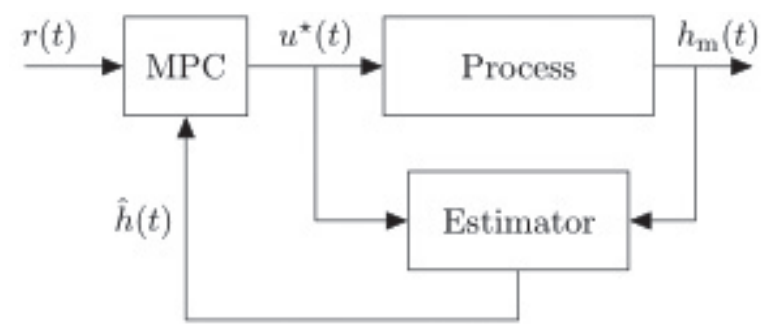

Fig. 2. General model predictive control strategy scheme: $r(t)$ stands for the reference signal, i.e. the desired liquid level, $u^{*}(t)$ is the optimal control action, i.e. the inlet flow of liquid. Actual measurement of the liquid level is depicted as $h_{\mathrm{m}}(t)$ while the estimate of the level is denoted as $\hat{h}(t)$.
The design and implementation of the model predictive controller follow the principles of the receding horizon policy established by Mayne et al. (2000). It optimizes control actions over the prediction horizon $N$ based on predictions of the future trajectory of the process variable.

\section{Non-linear MPC}

The non-linear model predictive controller was approached as an optimization problem with a quadratic cost function and non-linear equality constraints

$$
\min _{u_{0}, \ldots, u_{N-1}} \sum_{k=0}^{N-1}\left(\left.||\left(x_{k}-r_{k}\right)\right|_{Q_{\mathrm{x}}} ^{2}-\left.||\left(u_{k}-u_{k-1}\right)\right|_{Q_{\mathrm{u}}} ^{2}\right)
$$

s. t.

$$
\begin{gathered}
x_{\mathrm{k}+1=x_{\mathrm{k}}}+T_{\mathrm{S}} \cdot h\left(x_{\mathrm{k}}, u_{\mathrm{k}}\right), \\
h_{\text {min }} \leq x_{k} \leq h_{\text {max }}, \\
q_{\mathrm{in}, \min } \leq u_{k} \in q_{\mathrm{in}, \text { max }}, \\
\Delta q_{\mathrm{in}, \text { min }} \leq\left(u_{k}-u_{k-1}\right) \leq \Delta q_{\mathrm{in}, \text { max }}, \\
x_{0}=h(t), u_{-1}=u\left(t-T_{\mathrm{S}}\right) .
\end{gathered}
$$

The objective function in Eq. (13a) defines the difference between the predicted liquid level, $x_{\mathrm{k}}$, and the height reference, $r_{\mathrm{k}}$, followed by a second term which shows the increments of control actions. Such structure of the objective function enforces the offset-free control performance (Muske and Badgwell, 2002). Note that the term

$$
\|z\|_{M}^{2}=z^{\mathrm{T}} M z
$$

represents the squared Euclidean norm. The prediction equation (13b) is represented by the non-linear dynamic model in Eq. (5). Constraints presented in Eqs. (13c) and (13d) ensure that technological limits of the process variable and the manipulated variable are satisfied. Namely, constraint (13c) represents physical dimensions of the tank, constraint (13d) defines the range of inlet flow, while Eq. (13e) shows how fast the inlet flow can change, i.e., how fast can the control valve be opened or closed. Lastly, the optimization problem is initialized by the current measurement of height and by the previous control action as in Eq. (13f), and constraints $(13 \mathrm{~b})-(13 \mathrm{e})$ are valid for $k=0, \ldots$, $N-1$.

Remark 3.1 To prevent the infeasibility of the optimization problem given by Eq. (13), which can occur by introducing measurement noise or disturbances, the inequality constraint limiting the state variable can be decreased as suggested in (Primbs, 2007; Zeilinger et al., 2010). A slack variable was introduced to Eq. (13c), denoted by $s_{\mathrm{k}}$, as follows 


$$
-s_{k}+h_{\min } \leq x_{k} \leq h_{\max }+s_{k} .
$$

The slack variable was then defined in the objective function by adding the term

$$
\sum_{k=0}^{N-1}\left\|s_{k}\right\|_{Q_{s}}^{2} .
$$

The value of the penalization factor $Q_{s}$ should be chosen high compared to $Q_{\mathrm{x}}$ and $Q_{\mathrm{u}}$ to discourage the $N M P C$ controller from violating the constraint. Note that the slack variables $s_{\mathrm{k}}$ become additional optimization variables.

The optimization problem given by Eq. (13) can be solved by off-the-shelf tools like fmincon in Matlab, which employs optimization procedures like the interior-point method, trust-region or sequential quadratic programming (SQP) methods to obtain the optimal solution (Nocedal and Wright, 2006). To use the ready function fmincon, the optimization problem in Eq. (13) has to be rewritten to a standardized form

$$
\begin{gathered}
\min _{U} f(U) \\
\text { s. } \mathrm{t} c_{\mathrm{eq}}(U)=0, \\
c(U) \leq 0, \\
C_{\mathrm{eq}} U=b_{\text {eq }}, \\
C_{\text {ineq }} U \leq b_{\text {ineq }},
\end{gathered}
$$

where $U=\left[u_{0}, \ldots, u_{\mathrm{N}-1}\right]$ is the column vector of optimization variables. Then, the non-linear equality constraint (15b) is obtained from Eq. (13b), while the non-linear inequality constraint $(15 \mathrm{c})$ is derived from Eqs. (13b) and (13c). The linear constraints in Eqs. (15e) and (15d) are formed from Eqs. (13d), (13e), and (13f). The SQP method was suggested to solve the optimization problem since the objective function, Eq. (13), is a quadratic function.

\section{Linear-based MPC}

The linear version of MPC has the same structure as the non-linear version given by Eq. (13) except for the constraint (13b) which represents the prediction equation. Here, the non-linear dynamic equation is linearized by the Taylor first-order expansion at the operating point (cf. Remark 3.2) denoted as $\left(h_{\mathrm{L}}\right.$, $\left.q_{\text {in, } \mathrm{L}}\right)$. The resulting prediction equation has then the form of a linear state space model. A discretized version of the state space model with sampling time $T_{\mathrm{s}}$ is obtained

$$
x\left(t+T_{\mathrm{S}}\right)=A x(t)+B u(t)
$$

The state vector $x(t)$ and control input $u(t)$ are define as a deviation from the respective steady states values. Linear MPC was considered as a quadratic optimization problem (QP) with linear constraints. This QP problem can be then solved by quadprog function in Matlab or by the GUROBI solver. Note that the design of individual controllers is a general procedure, however, parameters from Table 1 were used to construct the optimization problems.

The optimization problem for linear MPC is formulated in the same fashion as described for the non-linear case, where the ready function fmincon was used. Here, however, Eqs. (15b) and (15c) were combined with Eq. (16) into (15d) and (15e), respectively, to obtain standardized quadratic optimization problem.

Remark 3.2 The operating point, often called "steady state", can be explicitly calculated from the non-linear model in Eq. (4) by solving

$$
\frac{1}{F\left(h_{L}\right)}\left(q_{i n, L}-k_{v} \sqrt{h_{L}}\right)=0 .
$$

Note that the choice of the operating point affects the performance of linear-based control strategies. Linearization point should be chosen with respect to technological properties of the plant.

\section{Comparisons and Results}

Both MPC controllers were designed with parameters reported in Table 1. The prediction horizon $N$ was set to 10 samples, which results in the prediction window of $20 \mathrm{~s}$. To fairly assess the performance of individual controllers, also the weighting factors were chosen to be identical for both controllers, i.e.,

$$
Q_{\mathrm{x}}=\frac{1}{h_{\mathrm{L}}^{2}} \text { and } Q_{\mathrm{u}}=\frac{5}{q_{\mathrm{L}}^{2}} .
$$

Additionally, the algorithm in fmincon was set to sequential quadratic programming with tolerance related to changes of the optimization variables to $10^{-8}$. Such settings ensured numerically sound solutions.

The performance of both controllers was compared for two simulation scenarios. The first scenario involved the tracking problem, where the ability of NMPC to control the liquid level to the highest limit was demonstrated. The second scenario involved a disturbance rejection problem with included measurement noise.

A simulation window of $400 \mathrm{~s}$ was considered for the tracking scenario, where two step changes on the reference value occurred at times $t_{\text {up }}=50 \mathrm{~s}$ and at $t_{\text {down }}=350 \mathrm{~s}$. The main benefit of the predictive controller can be seen exactly at these times where the controller reacts in anticipation of the reference change. Concrete time profiles are depicted in Fig. 3. Linear MPC is unable to control the liquid level to the highest limit since it would hit the con- 

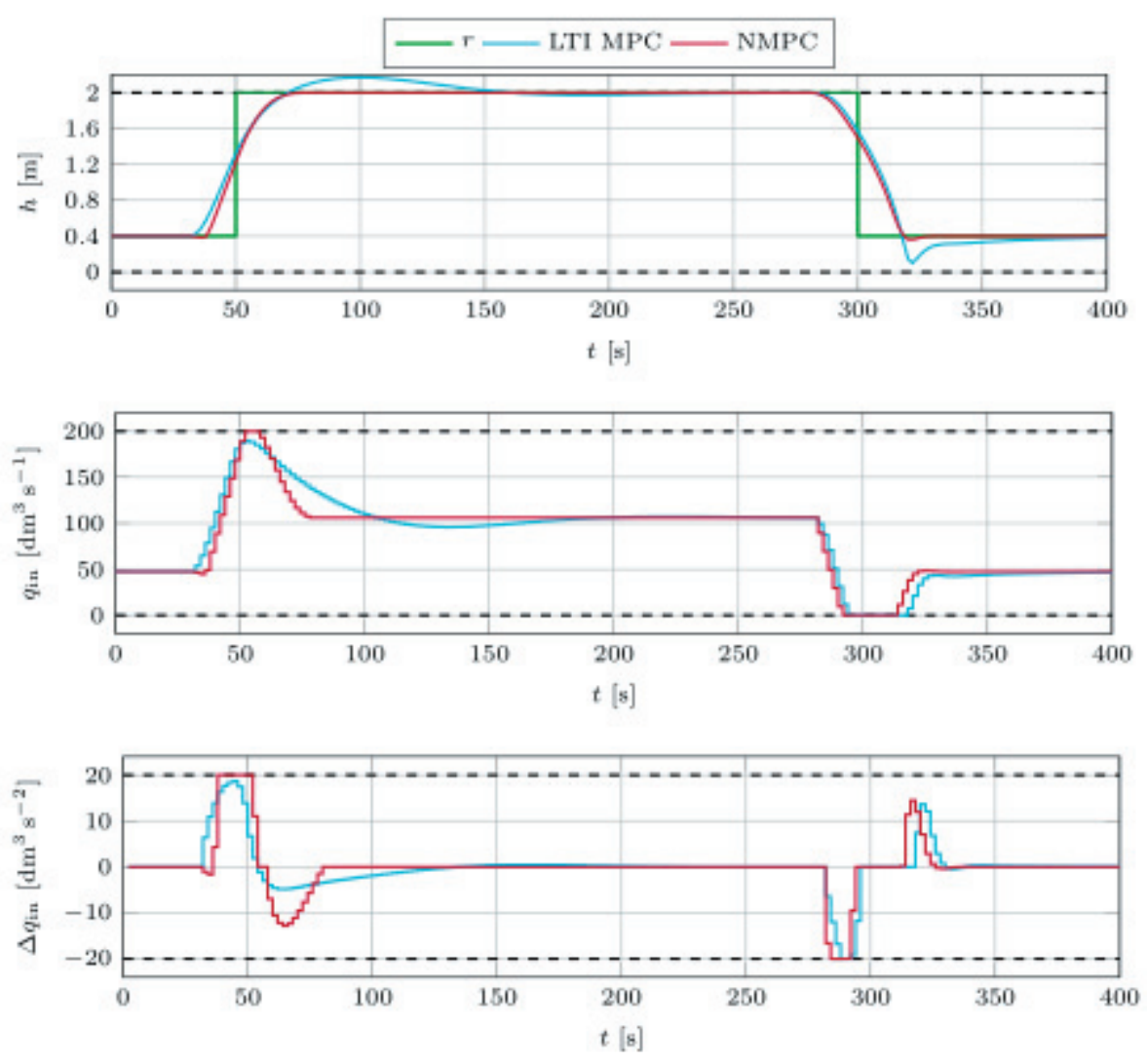

Fig. 3. Comparison of the control performance under linear-based MPC and non-linear model predictive control strategy. Simulation with linear MPC is possible only due to the softening of the constraints (cf. Remark 3.1).
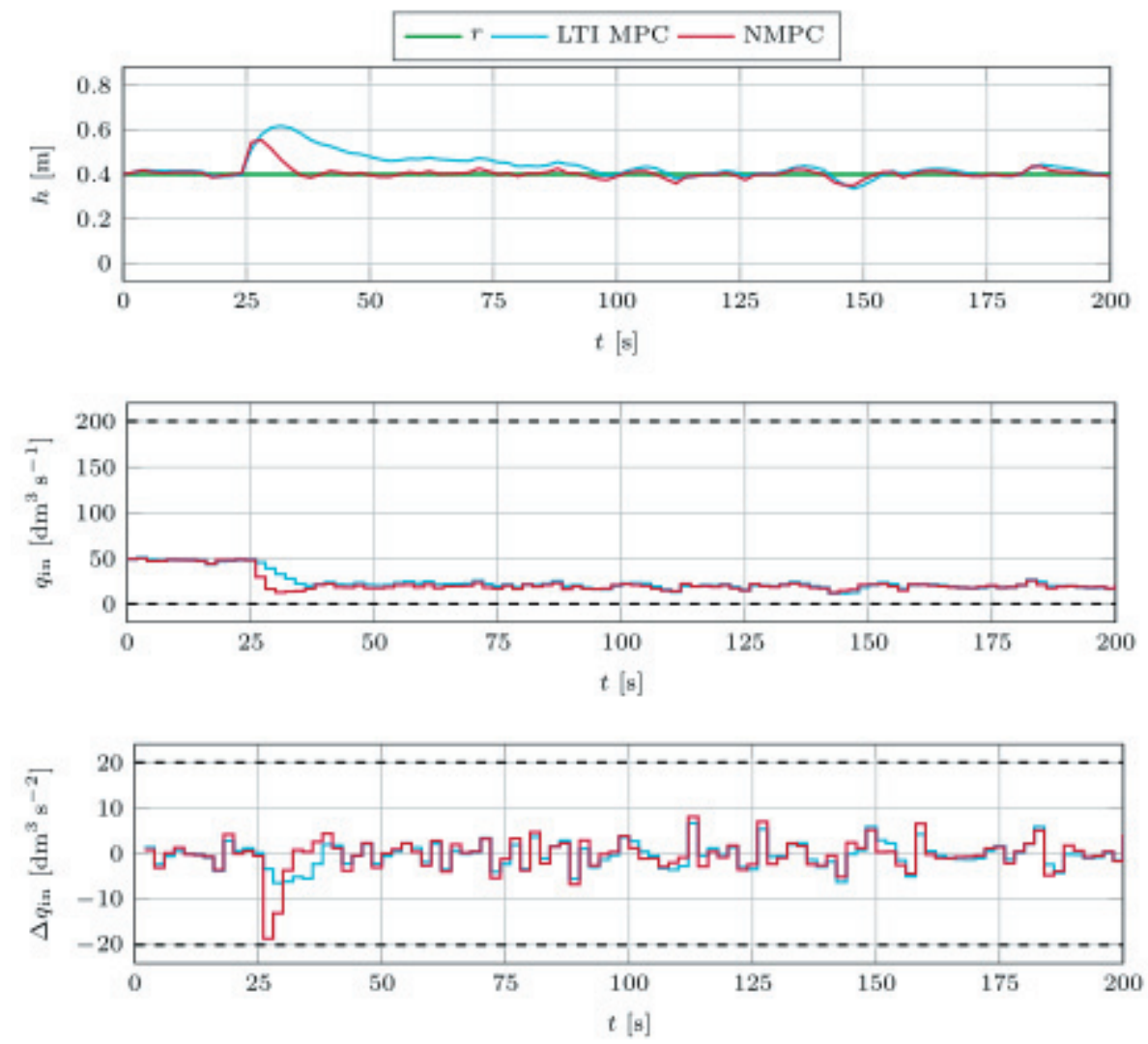

Fig. 4. Comparison of the performance of linear MPC and NMPC in the presence of disturbance and measurement noise. 
straint due to the overshoot and become infeasible. Similarly, a rather large undershoot occurs after time $t_{\text {down }}$.

Time profiles shown in Fig. 4 are related to the second scenario. Here, the performance of the NMPG strategy was tested rejecting disturbances, while measurement noise is present. An artificial input disturbance of $0.0285 \mathrm{~m}^{3} \mathrm{~s}^{-1}$ was introduced to the simulation around the time $t_{\mathrm{d}}=25 \mathrm{~s}$. Linear MPG requires considerably longer time to settle back to the reference value than NMPG. This behavior can be affected by the tuning factors $Q$ and $R$ in the Kalman filter design. On the other hand, placing higher weight to the disturbance estimation disrupts the estimation of unmeasured disturbances related to the model mismatch. This simulation scenario also includes the measurement noise, which was chosen as white noise with zero mean and standard deviation $\sigma=5 \cdot 10^{-2}$.

\section{Conclusions}

This paper covers the design of a non-linear predictive controller applied to control the liquid level inside a storage tank. The use of a control scheme involving the extended Kalman filter was proposed. Such a scheme ensures off-set free tracking of the reference even in the presence of disturbances. The non-linear predictive controller was implemented as an optimization problem with quadratic cost function with non-linear constraints in Matlab using the built-in function fmincon. The proposed NMPG strategy was then validated in a simulation of a conically shaped storage tank, which model was also derived. The NMPG strategy was compared with the well established linear-based MPC. Comparison made by simulation showed that NMPC outperforms the linear MPC in tracking as well as in rejecting disturbances.

\section{Acknowledgments}

The authors gratefully acknowledge the support of the Scientific Grant Agency of the Slovak Republic under the grant 1/0403/15, and the Research E Development Operational Programme for the project University Scientific Park STU in Bratislava, ITMS 26240220084, supported by the Research 7 Development Operational Programme funded by the ERDF. M. Klaučo would like to thank for the financial support from the $S T U$ in Bratislava Grant Scheme for Excellent Research Teams.

\section{References}

Al-Shahrani MH, Mahfoud M, Anvarbatcha R, Athar T, Asmari AA (2017) Pharmacognosy Communications 7: 34-40.

Allgöwer F, Findeisen R, Nagy ZK (2004) Nonlinear model predictive control: From theory to application.

Bakošová M, Oravec J (2014) Robust mpc of an unstable chemical reactor using the nominal system optimization. Acta Chimica Slovaca, 7(2): 87-93.

Camacho EF, Bordons C (2007) Model Predictive Control. Springer, $2^{\text {nd }}$ edition.

Jelemenský M, Klaučo M, Paulen R, Lauwers J, Logist F, Impe JV, Fikar M (2016) Time-optimal control and parameter estimation of diafiltration processes in the presence of membrane fouling. In 11th IFAC Symposium on Dynamics and Control of Process Systems, including Biosystems, volume 11, pages 242-247.

King M (2010) Process Control: A Practical Approach. Wiley.

Kvasnica M, Herceg M, Čirka L', and Fikar M (2010) Model predictive control of a cstr: A hybrid modeling approach. Chemical papers, 64(3): 301-309.

Lawryńczuk M (2017) Nonlinear predictive control of a boiler-turbine unit: A state-space approach with successive on-line model linearisation and quadratic optimisation. ISA Transactions, 67: 476-495.

Mayne DQ, Rawlings JB, Rao CV, Scokaert POM (2000) Constrained model predictive control: Stability and optimality. Automatica, 36(6): 789-814.

Mikleš J, Fikar M (2007) Process Modelling, Identification, and Control. Springer Verlag, Berlin Heidelberg.

Muske KR (1997) Steady-state target optimization in linear model predictive control. In American Control Conference, 1997. Proceedings of the 1997, volume 6, pages 3597-3601.

Muske KR, Badgwell TA (2002) Disturbance modeling for off set-freelinear model predictive control. Journal of Process Control, 12(5): 617-632.

Nocedal J, Wright SJ (2006) Numerical Optimization. Springer, New York, $2^{\text {nd }}$ edition.

Primbs J (2007) A soft constraint approach to Stochastic Receding Horizon Control. In Decision and Control, $200746^{\text {th }}$ IEEE Conference on, pages 4797-4802.

Rawlings JB, Mayne DQ (2009) Model predictive control: Theory and design.

Sharma A, Fikar M, Bakošová M (2015) Comparative study of time optimal controller with pid controller for a continuous stirred tank reactor. Acta Chimica Slovaca, 8(1): 27-33.

Zeilinger M, Jones C, Morari M (2010) Robust stability properties of soft constrained mpc. In Decision and Control (CDC), $201049^{\text {th }}$ IEEE Conference on, pages 5276-5282. 